\title{
FREQUENCY IMPLEMENTATION OF DISCRETE WAVELET TRANSFORMS
}

\author{
P.C. Tay and J.P. Havlicek \\ School of Electrical and Computer Engineering \\ University of Oklahoma, Norman, OK USA \\ Peter. C. Tayanoaa.gov, joebobaou . edu
}

\begin{abstract}
This paper implements the discrete wavelet transform in the discrete Fourier domain. The need for such an approach arose out of our desire to find a convenient means of realizing a new class of non-separable orientation selective 2-D wavelet filter banks that are designed directly in the DFT domain. The filter bank design process begins with a conventional separable 2-D perfect reconstruction parallel filter bank that is not orientation selective. In the DFT domain, each non-low pass channel is decomposed into the sum of two orientation selective frequency responses that are each supported on only two quadrants of the 2-D frequency plane. The resulting filter bank possesses the good joint localization properties of orthogonal wavelet transforms and offers both perfect reconstruction and orientation selectivity. However, the orientation selective channels are non-separable - they cannot be implemented as iterated 1-D convolutions according to the usual separable 2-D wavelet transform paradigm. To overcome this difficulty, we develop straightforward techniques for implemening the DWT directly in the DFT domain.
\end{abstract}

\section{INTRODUCTION}

It is well known that convolution of two signals in the time domain corresponds to pointwise multiplication of their respective Fourier transforms. Provided that the signal and/or filter is sufficiently large, multiplication in frequency generally offers a lower computational complexity than time domain convolution. Although we are not directly motivated here by concerns about computational efficiency, we do find that implementing the discrete wavelet transform (DWT) directly in the DFT domain results in a computationally efficient approach, especially when considering the DWT of long yet finite sequences or multi-dimensional signals including images and video.

The work of Daubechies has popularized the use of multi-resolution analysis and has connected quadrature mirror filter banks (QMF) to compactly supported continous

\footnotetext{
This work was supported in part by the National Science Foundation under grant DUE-01211596
}

functions for which appropriate sets of dilations and translations constitute a basis for $L^{2}(\mathbb{R})[1]$. The Mallat algorithm described in [2], a.k.a the DWT, has provided a method to construct multi-channel frequency selective filter banks. Both the 1-D and the separable 2-D DWT have been used in many signal and image processing algorithms. Most notably, the 2-D wavelet transform is used in the JPEG 2000 image compression standard [3]. Some recent methods for decomposing an image into oriented components can be found in $[4,5]$. Generally, such decompositions require a transform that is selective in both the magnitude of frequency and in the orientation.

Our motivation stems from the desire to create invertible AM-FM image transforms [6] to facilitate image processing in the modulation domain. Banks of densely spaced but nonorthogonal Gabor filters have traditionally been used for decomposing an image into locally coherent components prior to AM-FM demodulation. These filters are attractive because of their excellent joint localization properties and orientation selectivity. However, because such filterbanks cannot be made orthogonal, the possibility of an invertible AM-FM transform is precluded when Gabor analysis filters are used.

Alternatively, separable 2-D orthogonal wavelet transforms are attractive because they can provide perfect reconstruction. However, it is clear that they cannot provide orientation selectivity. So we set out to determine a filter bank structure that would provide perfect reconstruction, frequency selectivity, orientation selectivity, and good joint localization. What emerged was a simple and elegant frequency domain decomposition of the non-low pass channels in a separable 2-D filter bank into non-separable orientation selective channels without loss of the perfect reconstruction property. As a result of their genesis, such filter banks are very straightforward to implement directly in the DFT domain.

\section{DISCRETE WAVELET TRANSFORM}

A QMF consist of four half-band filters, the low pass analysis, the high pass analysis, the low pass synthesis, and the 
high pass synthesis filters are denoted as $\mathbf{f}_{a}, \mathbf{g}_{a}, \mathbf{f}_{s}$, and $\mathbf{g}_{s}$, resp. In the 1-D time domain the filtering process takes the form of convolving the sequence either linearly or circularly with the impulse responses of each filter. The outputs of the analysis filters are downsampled by a factor of two by discarding every other sample. Prior to filtering, i.e., convolving in time by the synthesis filters, the downsampled outputs of the analysis filters are upsampled by a factor of two by inserting zeros between every pair of samples. If the four filters $\mathbf{f}_{a}, \mathbf{g}_{a}, \mathbf{f}_{s}$ and $\mathbf{g}_{s}$ constitute a perfect reconstruction QMF, then the sum of the outputs of the synthesis filters is a delayed version of the input.

The action of the analysis filters can be implemented in the discrete Fourier domain as multiplication of the DFT of the $N$-point input sequence and the DFT of the analysis filters (zero padded to length $N$ ). Precisely, let $x[n]$ be any finite even length- $N$ sequence. If necessary, we append a zero to make $N$ even. Let $\widehat{f}_{a}[n]$ and $\widehat{g}_{a}[n]$ to be the low pass analysis and high pass analysis filters zero padded to length$N$, resp. We compute the output of the analysis filters prior to downsampling as pointwise multiplication of DFT's:

$$
\begin{aligned}
& Y[K]=X[k] \widehat{F}_{a}[k] \stackrel{D F T}{\longleftrightarrow} y[n], \\
& W[k]=X[k] \widehat{G}_{a}[k] \stackrel{D F T}{\longleftrightarrow} w[n],
\end{aligned}
$$

where $X[k], \widehat{F}_{a}[k], \widehat{G}_{a}[k]$ are the $N$-point DFT's of $x[n]$, $\widehat{f}_{a}[n], \widehat{g}_{a}[n]$, resp., and $n, k \in[0, N-1]$.

To downsample and subsequently upsample, let $\widehat{Y}[k]$ and $\widehat{W}[k]$ denote the analysis filter bank outputs downsampled by a factor of two and let $U[k], V[k]$ denote $\widehat{Y}[k], \widehat{W}[k]$ upsampled by a factor of two, resp. It is easy to verify the $z$-transform relation of the down sampling and subsequent upsampling as

$$
\begin{aligned}
U(z) & =\frac{1}{2}(Y(z)+Y(-z)) \\
V(z) & =\frac{1}{2}(Y(z)+Y(-z)) .
\end{aligned}
$$

The z-transforms (3) and (4) are easily converted into DFT's by equispaced sampling around the unit circle of the $z$ plane:

$$
\begin{gathered}
\widehat{Y}[k]=\frac{1}{2}\left(Y[k]+Y\left[\frac{N}{2}+k\right]\right), \\
\widehat{W}[k]=\frac{1}{2}\left(W[k]+W\left[\frac{N}{2}+k\right]\right),
\end{gathered}
$$

where $k \in\left[0, \frac{N}{2}-1\right]$. Thus $\widehat{y}[n]$ and $\widehat{w}[n]$ are length- $\frac{N}{2}$ sequences. The upsampling of $\widehat{y}[n]$ and $\widehat{w}[n]$ can be accounted for in the DFT domain by the following:

$$
U[k]= \begin{cases}\widehat{Y}[k] & \text { for } k \in\left[0, \frac{N}{2}-1\right] \\ \widehat{Y}\left[k-\frac{N}{2}\right] & \text { for } k \in\left[\frac{N}{2}, N-1\right]\end{cases}
$$

$$
V[k]= \begin{cases}\widehat{W}[k] & \text { for } k \in\left[0, \frac{N}{2}-1\right] \\ \widehat{W}\left[k-\frac{N}{2}\right] & \text { for } k \in\left[\frac{N}{2}, N-1\right] .\end{cases}
$$

We implement the synthesis filters in the DFT domain simply as the following multiplication

$$
\begin{aligned}
& X_{l}[k]=U[k] \widehat{F}_{s}[k], \\
& X_{h}[k]=V[k] \widehat{G}_{s}[k],
\end{aligned}
$$

where $\widehat{F}_{s}[k]$ and $\widehat{G}_{s}[k]$ are the DFT's of $f_{s}[n]$ and $g_{s}[n]$ after zero padding to length $N$, resp. Let $\widehat{X}[k]$ and $\widehat{x}[n]$ be a DFT pair, so that

$$
\widehat{X}[k]=X_{l}[k]+X_{h}[k] \stackrel{D F T}{\longleftrightarrow} \widehat{x}[n] .
$$

If $\mathbf{f}_{a}, \mathbf{g}_{a}, \mathbf{f}_{s}$, and $\mathbf{g}_{s}$ constitute a perfect reconstruction QMF, then $x[n]=\widehat{x}[(n+l) \bmod N]$ for some circular delay $l \in \mathbb{Z}$ and for all $n \in[0, N-1]$. The circular delay is determined by the length of the input sequence $(N)$ and by the original lengths of the filters $\mathbf{f}_{a}, \mathrm{~g}_{a}, \mathrm{f}_{s}$, and $\mathrm{g}_{s}$. For perfect reconstruction QMF's comprising length-six filters, the delay is accounted for according to

$$
l= \begin{cases}\frac{1}{2}|10-N| & \text { if } 6 \leq N \leq 10 \\ \frac{1}{2}(N+1-|1.0-N|) & \text { if } 10<N .\end{cases}
$$

The analysis filter bank and the synthesis filter bank can be cascaded to constitute a multi-level DWT. Fig. 1 shows the wavelet transform analysis filter bank cascaded to three levels. Fig. 2 shows the inverse wavelet transform synthesis filter bank cascaded to three levels. The Noble identities shown in Fig. 3 are stated as the following:

1. Down-sampling by $M$ then filtering by $G(z)$ is equivalent to filtering by $G\left(z^{M}\right)$ then down-sampling by $M$.

2. Filtering by $G(z)$ then up-sampling by $M$ is equivalent of up-sampling by $M$ then filtering by $G\left(z^{M}\right)$.

These identities allow the construction of an equivalent, parallel $L$-channel multi-rate analysis and synthesis filter bank. An arbitrary $L$-channel filter bank constructed from a $L-1$ level DWT is shown in Fig. 4. This construction yields $L$ analysis filters denoted as $H_{i}(z)$ and $L$ synthesis filters denoted as $\widetilde{H}_{i}(z)$ where $i \in[0, L-1]$. Though our construction applies to any positive integer $L$, we will use the case $L=5$ as an illustrative example. For a five-channel parallel filter bank which is equivalent to a four-level DWT, the Noble identities yield the following five analysis filters:

$$
\begin{aligned}
& H_{0}(z)=F_{a}(z) F_{a}\left(z^{2}\right) F_{a}\left(z^{4}\right) F_{a}\left(z^{8}\right), \\
& H_{1}(z)=F_{a}(z) F_{a}\left(z^{2}\right) F_{a}\left(Z^{4}\right) G_{a}\left(z^{8}\right), \\
& H_{2}(z)=F_{a}(z) F_{a}\left(z^{2}\right) G_{a}\left(z^{4}\right), \\
& H_{3}(z)=F_{a}(z) G_{a}\left(z^{4}\right), \\
& H_{4}(z)=G_{a}(z)
\end{aligned}
$$


the five synthesis filters are given by

$$
\begin{aligned}
\widetilde{H}_{0}(z) & =F_{s}\left(z^{8}\right) F_{s}\left(z^{4}\right) F_{s}\left(z^{2}\right) F_{s}(z), \\
\widetilde{H}_{1}(z) & =G_{s}\left(z^{8}\right) F_{s}\left(z^{4}\right) F_{s}\left(z^{2}\right) F_{s}(z), \\
\widetilde{H}_{2}(z) & =G_{s}\left(z^{4}\right) F_{s}\left(z^{2}\right) F_{s}(z), \\
\widetilde{H}_{3}(z) & =G_{s}\left(z^{2}\right) F_{s}(z) \\
\widetilde{H}_{4}(z) & =G_{s}(z) .
\end{aligned}
$$

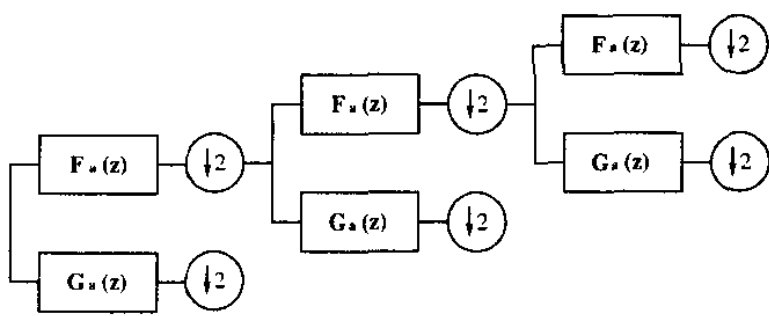

Fig. 1. The three-level discrete wavelet transform filter bank.

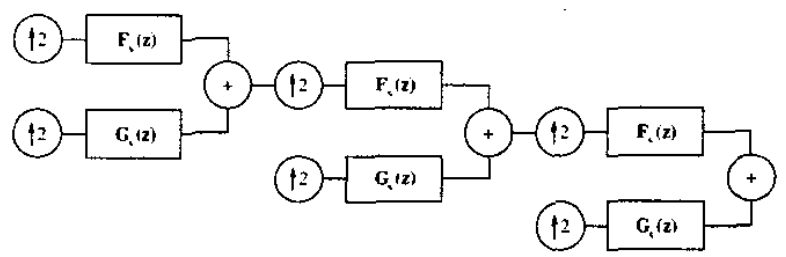

Fig. 2. The three-level inverse discrete wavelet transform filter bank.

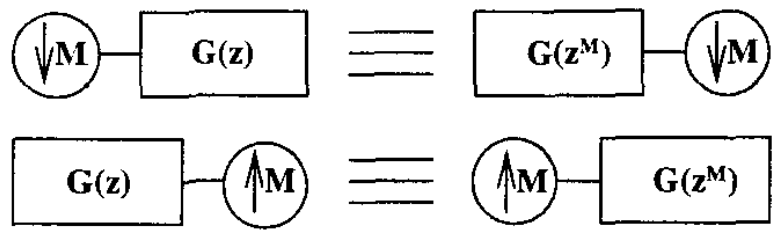

Fig. 3. The Noble identities.

An $L^{2}$-channel 2-D filter bank can be constructed separably from the analysis filters $H_{i}(z)$, where $i \in[0, L-$ 1]. Each 2-D filter is given by a product $\mathbf{H}_{i, k}[u, v]=$ $H_{i}(u) H_{k}(v)$, where $i, k \in[0, L-1]$ and $u, v \in[0, N-1]$. In our case, using a five-channel parallel filter bank, we get a twenty-five channel 2-D filter bank. This separable 2-D filter bank is magnitude frequency selective as shown by the $\log$ magnitude spectrum (LoMS) of the 2-D filters $\mathbf{H}_{0,0}=$ $H_{0}(u) H_{0}(v), \mathbf{H}_{0,1}=H_{0}(u) H_{1}(v), \mathbf{H}_{2,0}=H_{2}(u) H_{0}(v)$, and $\mathbf{H}_{3,3}=H_{3}(u) H_{3}(v)$ in Fig. 5. The LoMS of the appropriately zero padded separable $2-\mathrm{D}$ filters are shown in

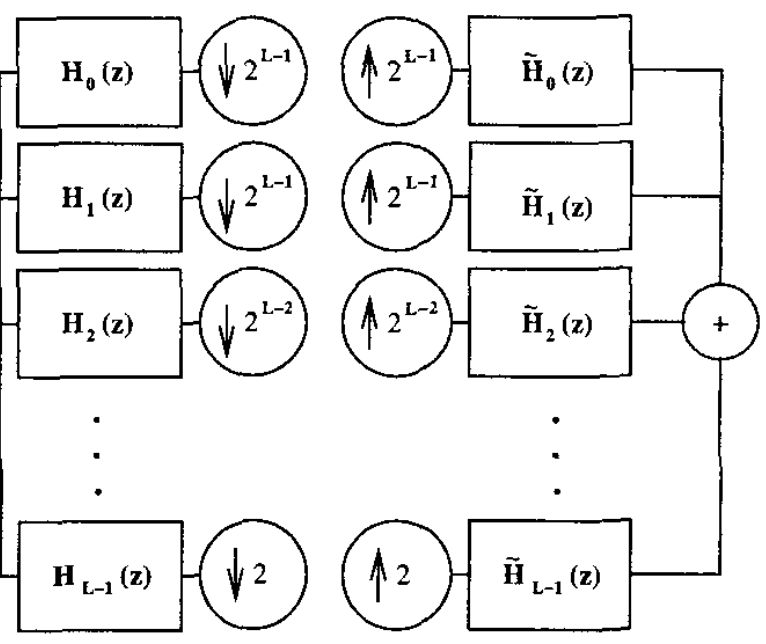

Fig. 4. A $L$-channel parallel filter bank.

Fig. 5, where they are frequency shifted so that the center of each image corresponds to origin of the 2-D frequency plane.

\section{ORIENTATION SELECTIVE FILTER BANK}

Though the 2-D filter bank created in the last section is frequency selective, it is not orientation selective. Orientation selectivity is important in many applications such as the AM-FM image model found in [6]. To achieve orientation selectivity, we decompose each non-low pass, nonorientation selective filter bank channel $\mathbf{H}_{i, k}, i, k \in[1, L]$, into a sum of two channels that are orientation selective, but are not separable. In decomposing the non-low pass 2D filters, it is necessary to consider the following theorem concerning the 1-D filter $H_{i}(z)$ in (14), (15), (16), and (17):

Theorem 1 If the high pass analysis filter $G_{a}(z)$ has a root at $z=1$, then the non-low pass filters $H_{1}(z), H_{2}(z), \ldots$, $H_{L-1}(z)$ of an $L$ channel parallel filter bank as shown in Fig. 4 exhibit zeros at $z=1$.

Proof: omitted for brevity.

From Theorem 1, it is easy to see that the LoMS of the 2-D separable filters $\mathbf{H}_{i, k}, i, k \in[1, L-1]$, are equal to zero on the hortizontal $(v=0)$ and vertical $(u=0)$ axes of the frequency plane. We partition the non-orientation selective channel filter $\mathbf{H}_{i, k}$ into a sum $\mathbf{H}_{i, k, t}+\mathbf{H}_{i, k, b}$ of nonseparable orientation selective channels according to

$$
\mathbf{H}_{i, k, t}= \begin{cases}\mathbf{H}_{i, k} & \text { for quadrants I and III } \\ 0 & \text { for quadrants II and IV }\end{cases}
$$

and

$$
\mathbf{H}_{i, k, b}= \begin{cases}\mathbf{H}_{i, k} & \text { for quadrants II and IV } \\ 0 & \text { for quadrants I and III. }\end{cases}
$$




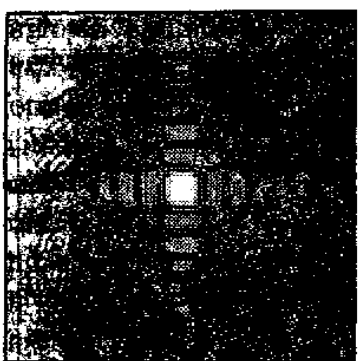

(a) LoMS of $\mathbf{H}_{0,0}$.

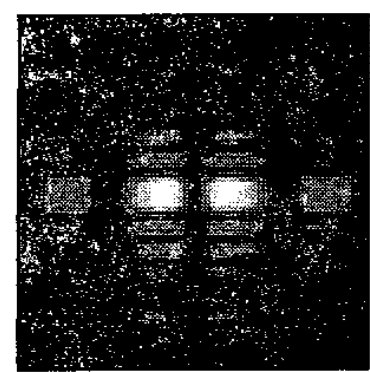

(c) LoMS of $\mathbf{H}_{2,0}$.

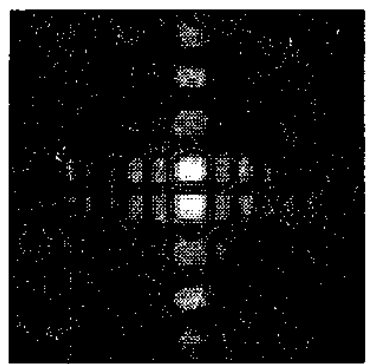

(b) LoMS of $\mathbf{H}_{0,1}$.

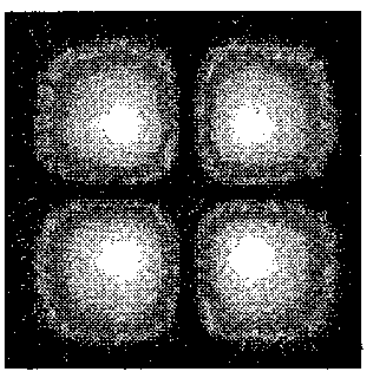

(d) LoMS of $\mathbf{H}_{3,3}$.
Fig. 5. Log Magnitude Spectra

Fig. 6 shows the LoMS of $\mathbf{H}_{3,3, t}$ and $\mathbf{H}_{3,3, b}$. This increases the number of analysis channels from twenty-five to fourtyone, and the resulting filters can no longer be implemented separably. However, we have achieved both orientation selectivity and perfect reconstruction. (a) LoMS of $\mathbf{H}_{3,3, t}$.

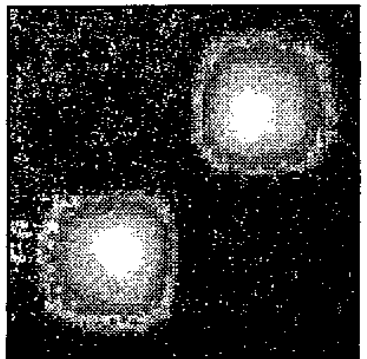

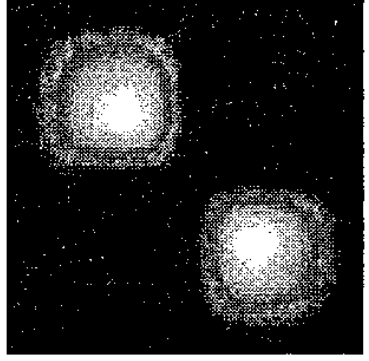

(b) LoMS of $\mathbf{H}_{3,3, b}$.
Fig. 6. $\mathbf{H}_{3.3}$ decomposed into two orientation selective channels.

\section{RESULTS}

In this section, we show the outputs of several frequency and orientation selective analysis filters constructed using the techniques given in this paper. Though any "regular" wavelet can be used in the QMF composition and subse- quent $L$-channel parallel filter bank, the QMF used in this section is the well-know Daubechies orthogonal length sixwavelet filter bank. The high pass analysis filter in this QMF satisfies the conclition of Theorem 1. Thus the decomposition of the non-low pass 2-D channels is possible. We use the common $256 \times 256$ Lena image to show that the filters provide the necessary selectivity. All filtering is implemented directly in the frequency domain. After the non-low pass 2-D filters are decomposed into orientation selective channels, the 2-D DFT of the Lena image is multiplied pointwise with each filter frequency response. The original Lena image is shown in Fig. 7(a). Fig. 7(b) gives the output of the non-orientation selective filter $\mathrm{H}_{3,3}$. Responses of the orientation selective filters $\mathbf{H}_{3,3, t}$ and $\mathbf{H}_{3,3,6}$ are shown in Fig. 7(c) and (d). In Fig. 7 (b), the response of the non-orientation selective filter $\mathbf{H}_{3,3}$ clearly exhibits a combination of noncoherent features from multiple orientation; this is highly undesirable in AM-FM image modeling and a number of other applications. As demonstrated by Fig. 7, this problem is effectively solved by decomposing the $\mathbf{H}_{3.3}$ filterbank channel into a pair of equivalent orientation selective channels.

Fig. 8 (a) and (b) show the responses of the nonorientation selective filters $\mathbf{H}_{1,2}$ and $\mathbf{H}_{2,1}$, resp. These two figures exhibit many instances of noncoherent features that intersect at perpendicular angles. Fig. 9 (a)-(d) depict the responses obtained by filtering the Lena image with the orientation selective filters $\mathbf{H}_{1,2, t}, \mathbf{H}_{1,2, b}, \mathbf{H}_{2,1, t}$, and $\mathbf{H}_{2,1 . b}$, resp.

Reconstruction of the original image is straightforward, except that the relationship of the circular delay of the cascaded system is not evident when implementing the tranform as an equivalent parallel filter bank realization. Resolving this issue will require future work in number theory and is beyond the scope of this paper.

\section{CONCLUSION AND FUTURE WORK}

In this paper, we began by describing an algorithm to implement a general QMF in the DFT domain. As with time domain implementations, the frequency domain QMF can be cascaded at multi-levels as originally described by Mallat. This frequency domain DWT implementation could potentially be exploited to realize a fast frequency domain wavelet transform algorithm. However, this was not our motivation here and we did not concern ourselves with recording the number of floating point calculations required.

Instead, our interest was in developing a 2-D perfect reconstruction filter bank with orientation selectivity and good join localization properties. The filterbank design we presented achieves this, but at the expense of separability. Nevertheless, this design admits an elegant and straightforward frequency domain implementation. Our experimental 


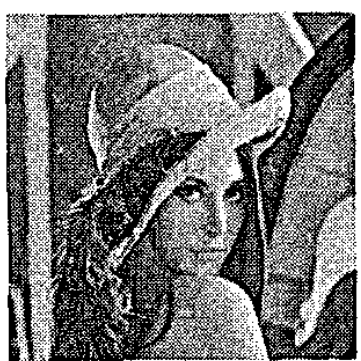

(a) Original

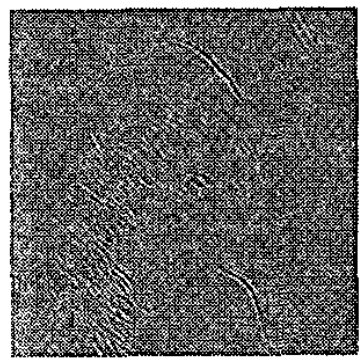

(c) Output of channel $\mathbf{H}_{3,3, t}$.

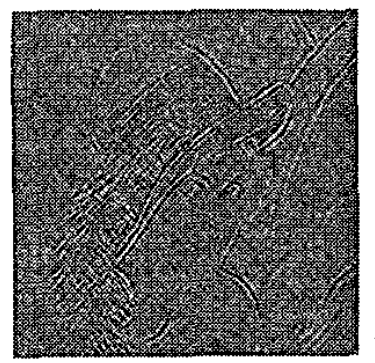

(b) Output of channel $\mathbf{H}_{3,3}$.



(d) Output of channel $\mathbf{H}_{3,3, b}$.

Fig. 7. Original and filtered Lena images.

results demonstrated dramatically the orientation selectivity of a non-separable wavelet filter bank obtained by frequency domain decomposition of a standard Daubechies length six separable 2-D orthogonal QMF.

In closing, we note that this approach of decomposing a separable but non-orientation selective filter bank structure into a new structure that is orientation selective but nonseparable is not restricted to the DWT.



(a) Output of $\mathbf{H}_{1,2}$

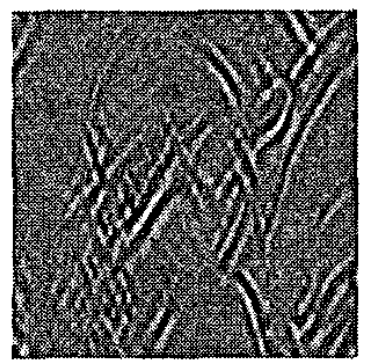

(b) Output of $\mathbf{H}_{2,1}$.

Fig. 8. Lena filtered by two non-orientation selective filters.

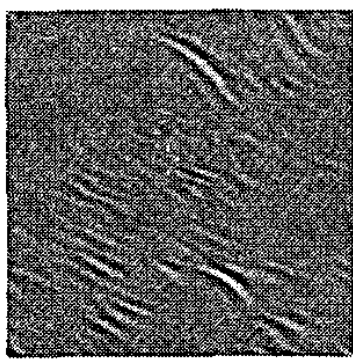

(a) Output of $\mathbf{H}_{1,2, t}$.

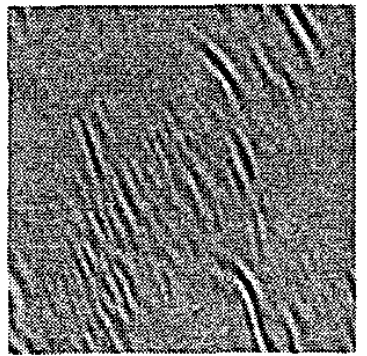

(c) Output of $\mathbf{H}_{2,1, t}$.

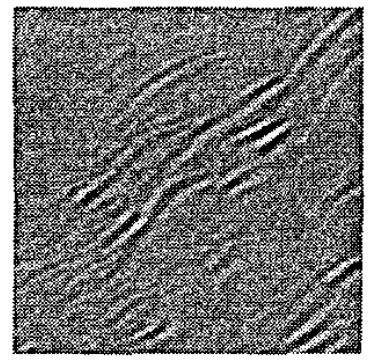

(b) Output of $\mathbf{H}_{1,2, b}$.



(d) Output of $\mathrm{H}_{2,1, b}$.
Fig. 9. Various filtered Lena image.

\section{REFERENCES}

[1] I. Daubechies, "Orthonormal bases of compactly supported wavelets," Commun. Pure Appl. Math., vol. 51, pp. 909-996, 1988.

[2] S. G. Mallat, "A theory for multiresolution signal decomposition: the wavelet representation," IEEE Trans. Pattern Anal. Machine Intell., vol. 11, no. 7, pp. 674693, July 1989.

[3] S. Lawson and J. Zhu, "Image compression using wavelets and JPEG2000: a tutorial," Electronics \& Communication Engineering Journal, vol. 14, no. 3, pp. 112-121, 2002.

[4] M. N. Do and M. Vetterli, "Contourlets: A directional multiresolution image representation," in Proc. IEEE Int'l. Conf. Image Proc., Rochester, NY, Sept. 22-25, 2002, pp. 589-592.

[5] V. Velisavljevic, P. L. Dragotti, and M. Vetterli, "Directional wavelet transforms and frames," in Proc. IEEE Int'l. Conf. Image Proc., Rochester, NY, Sept. 22-25, 2002, pp. 357-360.

[6] J. P. Havlicek, D. S. Harding, and A. C. Bovik, "Multidimensional quasi-eigenfunction approximations and multicomponent AM-FM models," IEEE Trans. Image Proc., vol. 9, no. 2, pp. 227-242, February 2000. 